\title{
THE ULYSSES MISSION IN
}

\section{THE HIGH LATITUDE HELIOSPHERE}

\author{
D.E. PAGE \\ ESA Ulysses Office, Jet Propulsion Laboratory, Pasadena, CA 91109, USA \\ R.G. MARSDEN \\ Space Science Department, ESTEC, 2200 AG Noordwijk, Netherlands \\ E.J. SMITH \\ Jet Propulsion Laboratory, California Institute of Technology, Pasadena, CA 91109, USA \\ and \\ K.-P. WENZEL \\ Space Science Department, ESTEC, 2200 AG Noordwijk, Netherlands
}

\begin{abstract}
Ulysses, a joint ESA/NASA mission launched in October 1990, will be the first to explore the high latitude heliosphere. Launch will be from the Shuttle and a Jupiter gravity assist will be used to send the spacecraft first over the southern solar pole approximately three and one half years after launch and then over the northern solar pole one year later. Instruments will be carried to study the solar wind, the heliospheric magnetic field, energetic solar particles, galactic cosmic rays, solar $\mathrm{X}$-rays, cosmic gamma rays, cosmic dust and interstellar neutral helium. The radio signals used to track and transmit spacecraft data will be used also to sound the corona and to search for gravitational waves.
\end{abstract}

\section{Introduction}

All spacecraft to date have remained fairly close to the ecliptic plane. Ulysses, a joint ESA/NASA mission launched in October 1990, will be the first to explore the heliosphere at high solar latitudes. Measurements of the solar wind, the interplanetary magnetic field and high energy solar particles have been carried out since the beginning of the space age but almost certainly the results obtained are applicable only to a narrow belt of helio- latitude close to the ecliptic plane. (Marsden (1986) and references therein, Page (1975)). The solar atmosphere is clearly non-uniform with, for example, flare sites progressing through the latitude range 30 to 5 degrees as the solar cycle progresses. Various observations, including the relationship between geomagnetic activity and the position of activity on the solar disk, lead us to believe that the solar wind blows more or less radially from the sun. To sample what leaves the sun at a particular latitude it is therefore necessary to make in-situ measurements of the solar atmosphere at that latitude.

\section{Some Science Goals}

\subsection{The Solar Wind}

There is good reason to believe that the solar wind flow pattern shows characteristic latitude differences. The equatorial regions are dominated by the complex streamstream interactions caused by solar rotation and a characteristic variation of solar

Y. Kondo (ed.), Observatories in Earth Orbit and Beyond, 307-313.

(C)1990 Kluwer Academic Publishers. Printed in The Netherlands. 
wind speed with longitude. At high latitudes, the flow speed is expected to be higher, more uniform and nearly parallel to the magnetic field. Here it may be easier to understand the evolution of the solar wind with distance and to relate its properties to conditions in the solar source region.

The configuration of the Sun and heliosphere evolves throughout the solar cycle. During solar minimum conditions, coronal holes extend equatorwards from the poles to form large, well-defined open magnetic field regions. The corona then exhibits well-developed equatorial streams and departs significantly from spherical symmetry. High speed solar wind streams are observed at low latitudes and unipolar regions form to the north and south of the nearly flat equatorial current sheet. At solar maximum, such long-lasting coronal holes are generally absent and the pattern of solar wind streams and the heliospheric magnetic field becomes less ordered. The polarity of the Sun reverses about a year after solar maximum, leading to a major re-structuring of the heliosphere. There is a possibility that multiple current sheets occur at high latitudes.

\subsection{The Heliospheric Magnetic Field}

The heliospheric magnetic field is known to vary with latitude. In equatorial regions the field is wound into a spiral by the rotation of the Sun and at large distances becomes azimuthal. In polar regions the influence of solar rotation decreases and presumably the field becomes radial and weaker. (Models indicate that the radial field falls off as $R^{-2}$ whereas the azimuthal component decreases as $R^{-1}$ ). Jokipii and Kota (1989) however suggest that large transverse field fluctuations can be expected in polar regions.

\subsection{Energetic Charged Particles from the Sun}

It is unclear whether energetic particles accelerated in active regions on the sun can propagate to high latitudes. Solar flare particles may originate and propagate primarily in the complex magnetic field structures at low solar latitudes, but their source regions and/or propagation may also extend to considerably higher latitudes, at least during part of the solar cycle. Interplanetary acceleration processes, presumably related to interplanetary shocks, have been shown to occur at least up to the highest heliographic latitude reached by Voyager $1\left(38^{\circ}\right)$. Ulysses will extend observations to cover the full range of heliolatitudes.

\subsection{The Cosmic Radiation}

The solar wind and the frozen-in magnetic field carried by it prevent a part of the cosmic radiation from penetrating to the inner heliosphere. Knowledge of the primary cosmic ray spectrum and composition outside the heliosphere is something which has been sought for many years. If the heliospheric magnetic field at high latitudes is indeed essentially radial, it may be that the cosmic radiation arriving over the solar poles is unmodulated by the solar field and is therefore more representative of the cosmic radiation in the local interstellar medium. There is an almost complete lack of information on how galactic cosmic rays are modulated at 
high solar latitudes. Measurements of the flux variation with latitude are therefore particularly interesting. Extrapolation to high heliographic latitudes of our present understanding of cosmic ray transport in the heliosphere, derived from observations near the heliospheric equator, suggests that the particle gradient and curvature drifts in the large-scale magnetic field and heliospheric current sheet may play an important role in cosmic ray access to the heliosphere and solar modulation (e.g. McKibben 1986, Smith and Thomas 1986). If drifts are important, then following the next solar field reversal (to positive magnetic field in the northern hemisphere, expected in 1991/92), the net flow will change from outward to inward over the solar poles for positively charged particles. Thus a definite latitude variation of ion intensities should be detected, with the opposite behaviour expected for electrons.

Most of the instrumentation on Ulysses is designed to study features of solar wind, the heliosphere magnetic field and energetic charged particles (Table 1.)

Further instrumentation is designed to study:

(a) Solar x-rays which generally accompany the production of high energy electrons at the sun.

(b) Cosmic gamma-rays. The Ulysses measurements will be combined with measurements from other spacecraft in an attempt to pin-point the origin of cosmic gamma bursts.

(c) Radio and Plasma Waves. These may be produced at the sun, by interactions in the solar wind and as electrons spiral about the interplanetary field.

(d) Cosmic Dust. Investigation of the physical and dynamical properties of the dust as a function of heliocentric distance and ecliptic latitude should help determine the relative significance of comets, asteroids and meteors as dust sources.

(e) Interstellar Neutral Helium. The solar system moves through the interstellar gas at about $20 \mathrm{~km} / \mathrm{s}$. Neutral helium entering follows Keplerian orbits in the gravitational field of the sun. Measurements made at the widely separated points which are not co-planar with respect to the symmetry axis of the helium flow direction should permit determination of arrival velocity.

Two other investigations make use of the radio signals which are supplied to transmit data and to track the spacecraft.

(f) Coronal Sounding. When the radio signals from the X and S band transmitters pass through the solar corona en route from spacecraft to earth it should be possible to determine the total electron content through which the radio waves have passed and to follow fluctuations in electron density.

(g) Gravitational Waves. A gravitational wave produced for example by a violent event in a galactic nucleus may perturb the spacecraft orbit sufficiently to be seen in the tracking Doppler data. A measurement accuracy of around $3 \times 10^{-14}$ is required.

A detailed description of the scientific instruments can be found in Wenzel et al. (1983).

\section{Spacecraft Trajectory}

Ulysses will be launched in the ecliptic plane toward Jupiter by a combined twostage IUS/PAM-S upper stage solid rocket motor, carried on board the NASA space shuttle. The Jovian gravity field will deflect the suitably directed spacecraft 


\section{Investigation/ Principal Investigator}

Magnetic Fields (HED)
A. Balogh,

Imperial College, London, UK

Solar Wind Plasma (BAM)

S. J. Bame,

Los Alamos Nat Lab, USA

Solar Wind Ion

Composition (GLG)

G. Gloeckler,

U. Maryland, USA

J. Geiss

U. Bern. $\mathrm{CH}$

Low Energy Ions

and Electrons (LAN)

L. Lanzerotti

Bell Laboratories, USA

Energetic Ion Composition

and Interstellar Gas (KEP)

E. Keppler,

MPAe, Lindau, FRG

Cosmic Rays and Solar Particles

(SIM)

J. A. Simpson,

U. Chicago, USA
Collaborating Institutes

Magnetic fields:

JPL

$\pm 0.01- \pm 44000 \mathrm{nT}$

Ions: $257 \mathrm{eV} / \mathrm{Q}-35 \mathrm{keV} / \mathrm{Q}$

$\mathrm{e}^{-} \quad: 1--903 \mathrm{eV}$

NASA/ARC

JPL

HAO, Boulder

UCLA

NASA/MSFC

MPAe, Lindau

Ion composition:

$145 \mathrm{~km} / \mathrm{s}(\mathrm{H})-$

$1352 \mathrm{~km} / \mathrm{s}\left(\mathrm{Fe}^{+8}\right)$

NASA/HQ

NASA/GSFC

TU Braunschweig

U. Natal

MPAe, Lindau

Ions: $50 \mathrm{keV}--5 \mathrm{MeV}$

UCB

e-: $30--300 \mathrm{keV}$

ion composition:

$0.2-15 \mathrm{MeV} / \mathrm{n}(\mathrm{Fe})$

U. Kansas

APL

Obs. Paris

U. Thrace

U. Birmingham

Ion Composition:

$80 \mathrm{keV}-15 \mathrm{MeV} / \mathrm{n}$

Neutral He

Imperial College Geophys. Obs. Kiruna

MPI, Heidelberg Aerospace Corp. U. Bonn

Nuclei: 0.3 - $600 \mathrm{MeV} / \mathrm{n}$ e-: $1-2000 \mathrm{MeV}$

\author{
Imperial College \\ ESA/SSD \\ NRC, Canada \\ U. Kiel \\ CEN, Saclay \\ Danish Sp. Res. \\ Inst. \\ NRC, Milan \\ MPI, Heidelberg \\ U. Maryland \\ MPAe, Lindau
}


TABLE I (continued)

Radio and Plasma Waves (STO)

R. G. Stone

NASA/GSFC, USA

Solar X-Rays and

Cosmic $\gamma$-Ray Bursts (HUS)

K. Hurley,

Space Sciences Lab,

Berkeley, USA and CESR, Toulouse, F.

M. Sommer,

MPI Garching, FRG

Cosmic Dust (GRU)

E. Grün,

MPI, Heidelberg, FRG

\section{Radio Science/}

Coronal Sounding (SCE)

H. Volland,

U. Bonn, FRG

Radio Science/

Gravitational Waves (GWE)

B. Bertotti

U. Pavia, I

Interdisciplinary/

Directional Discontinuities

J. Lemaire,

Inst. d'Aeronomie Spatiale

de Belgique, Brussels, B

Interdisciplinary/Mass Loss and Ion

Composition

G. Noci,

Obs. Arcetri, Florence, I
Plasma Waves: $0-60 \mathrm{kHz}$

Radio: $1--940 \mathrm{kHz}$

Magnetic field: $10--500 \mathrm{~Hz}$

$\mathrm{X}-, \gamma$-rays: $5--150 \mathrm{keV}$

Dust Particles

$10^{-16}-10^{-9} \mathrm{~g}$

Spacecraft dual-frequency ranging and Doppler data

Spacecraft Doppler data
Obs. Paris

U. Minnesota

CNET/CRPE, St.

Maur

Obs. Paris

SLR, Ultrecht

NASA/GSFC

CESR, Toulouse
U. Canterbury NASA/JSC

U. Bochum

ESA/SSD

U. Bochum

CNR, Frascati U. Uppsala JPL

Aerospace Corp. 


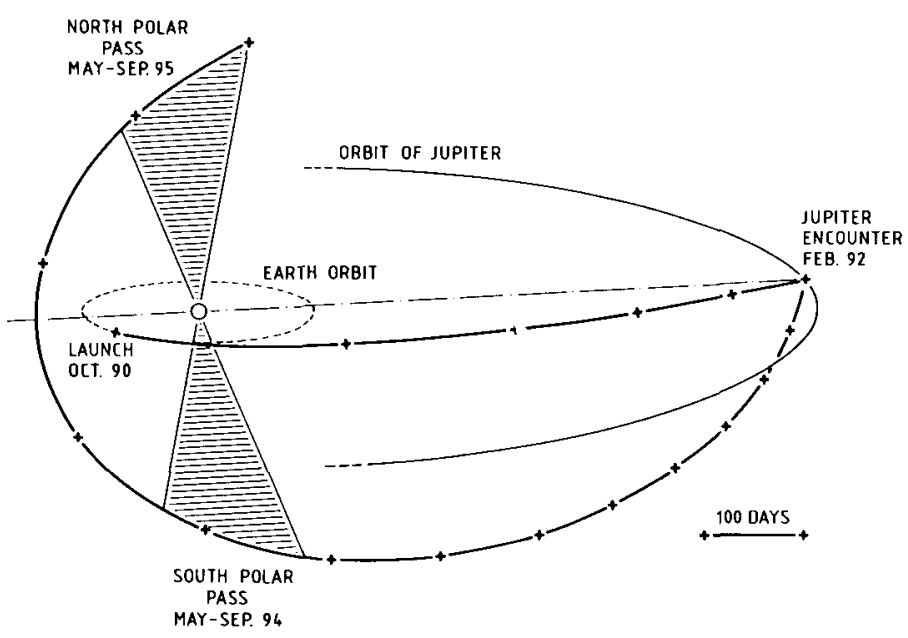

Fig. 1. Typical ULYSSES trajectory viewed from $15 \mathrm{deg}$ above the ecliptic plane. Crosses are shown at 100-day intervals.

so that it travels southward from the ecliptic plane to pass over the south solar pole about $3-1 / 2$ years after launch. At this point Ulysses will be approximately 2.3 $\mathrm{AU}$ below the ecliptic. Perihelion at $1.4 \mathrm{AU}$ will be reached about 6 months later still and nominal end of mission will arrive following the north solar polar pass in September 1995. The trajectory is illustrated in Figure 1. Launch in October 1990 (the window is 18 days long) will mean that most of the time spent at high solar latitudes should occur during a fairly quiet part of the solar cycle. This should mean that heliospheric structure will be relatively stable and well ordered and that it will be less difficult to differentiate between genuine latitude variations and random time variations.

\section{Operations and Data Recovery}

Ulysses will be tracked by NASA's Deep Space Network, operations being directed by a small ESA team resident at the Jet Propulsion Laboratory in Pasadena. Real time data will be transmitted at 1024 bits per second for 8 hours each day. During the remaining 16 hours, data continuity will be assured by using on board tape recorders which will record scientific data at 512 bits per second.

Tapes (Experiment Data Records) of cleaned and sorted data will be provided by JPL to each Principal Investigator, who carries the responsibility to pass on the relevant data to his co- investigators. A Common Data Record will also be prepared to assist data correlation between instruments and to help identify when events of special note occurred. This Record contains selected channels of relatively raw data extracted from each of the main experiments carried on the spacecraft. 


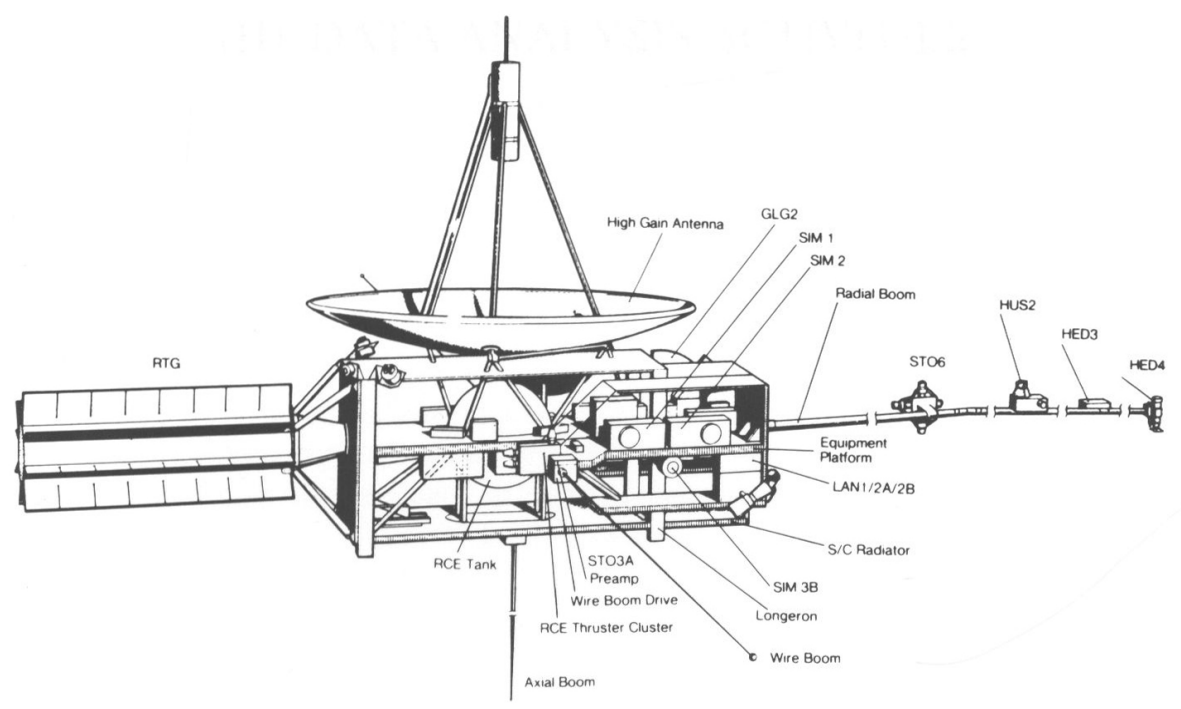

Fig. 2. Schematic of the ULYSSES spacecraft. The experiment codes are listed in Table 1: the number following the code identifies the sub-unit of a specific experiment

\section{The Spacecraft}

The spacecraft layout is illustrated in Figure 2. It measures about 3 meters across and about 2 meters in height with booms folded. At launch it will weigh approximately $370 \mathrm{~kg}$ including the $55 \mathrm{~kg}$ of scientific instrumentation. Because Ulysses must operate out to $5.4 \mathrm{AU}$ from the sun, power is obtained from a Radioisotope Thermoelectric Generator (RTG) which will provide close to 290 watts at launch. The spacecraft will be spin stabilized at $5 \mathrm{rpm}$ with information for logic operations coming from a sun sensor. At some parts of the trajectory, it will not be easy to keep the solar aspect angle above the minimum acceptable and at the same time to point the high gain parabolic antenna at the earth. S band telemetry is used for the up-link and both $\mathrm{S}$ and $\mathrm{X}$ band for the down-link. $\mathrm{S}$ band is at $2.3 \mathrm{GHz}$ and $\mathrm{X}$ band at $8.4 \mathrm{GHz}$.

\section{References}

Jokipii, J.R. and J. Kota: 1989, 'The polar heliospheric magnetic field', J. Geophys.Res., 16

Marsden, R.G.: 1986, The Sun and the Heliosphere in Three Dimensions, Reidel, Dordrecht McKibben, R.B.: 1986, in R.G. Marsden, ed(s)., The Sun and The Heliosphere in Three Dimensions, Reidel, Dordrecht, 361

Page, D.E.: 1975, Science 190, 845

Smith, E.J. and B. Thomas: 1986, J. Geoph. Res. 91, 2933

Wenzel, K.-P., R.G. Marsden and B. Battrick, 1983, The International Solar Polar Mission - Its Scientific Objectives, European Space Agency SP-1050 